\title{
Analysis of Handling Qualities Design Criteria for Active Inceptor Force-Feel Characteristics
}

\author{
Carlos A. Malpica \\ NASA Ames Research Center \\ Moffett Field, CA \\ Jeff A. Lusardi \\ Aeroflightdynamics Directorate (AMRDEC) \\ U.S. Army RDECOM \\ Moffett Field, CA
}

\begin{abstract}
\section{Background}

The force-feel system characteristics of the cyclic inceptors of most helicopters are set based on the characteristics of the mechanical components in the control system (mass, springs, friction dampers, etc.). For these helicopters, the force-feel characteristics typically remain constant over the entire flight envelope, with perhaps a trim release to minimize control forces while maneuvering. With the advent of fly-by-wire control systems and active inceptors in helicopters, the force-feel characteristics are now determined by the closed-loop response of the active inceptor itself as defined by the inertia, force/displacement gradient, damping, breakout force and detent shape configuration parameters in the inceptor control laws. These systems give the flexibility to dynamically prescribe different feel characteristics for different control modes or flight conditions, and the ability to provide tactile cueing to the pilot through the actively controlled side-stick or center-stick cyclic inceptor.
\end{abstract}

For rotorcraft, a few studies have been conducted to assess the effects of cyclic force-feel characteristics on handling qualities in flight. An early study provided valuable insight into the static force-deflection characteristics (force gradient) and the number of axes controlled by the side-stick controller for the U.S. Army's Advanced Digital/Optical Control System (ADOCS) demonstrator aircraft [1]. The first of a series of studies providing insight on the inceptor dynamic force-feel characteristics was conducted on the NASA/Army CH-47B variable-stability helicopter [2]. This work led to a proposed requirement that set boundaries based on the cyclic natural frequency and inertia, with the stipulation of a lower damping ratio limit of 0.3 [3]. A second study was conducted by the Canadian Institute for Aerospace Research using their variable-stability Bell 205A helicopter [4]. This research suggested boundaries for stick dynamics based on natural frequency and damping ratio. While these two studies produced boundaries for acceptable/unacceptable stick dynamics for rotorcraft, they were not able to provide guidance on how variations of the stick dynamics in the acceptable region impact handling qualities.

More recently, a ground based simulation study [5] suggested little benefit was to be obtained from variations of the damping ratio for a side-stick controller exhibiting high natural frequencies (greater than $17 \mathrm{rad} / \mathrm{s}$ ) and damping ratios (greater than 2.0). A flight test campaign was conducted concurrently on the RASCAL JUH-60A in-flight simulator and the ACT/FHS EC-135 in flight simulator [6]. Upon detailed analysis of the pilot evaluations the study identified a clear preference for a high 
damping ratio and natural frequency of the center stick inceptors. Side stick controllers were found to be less sensitive to the damping.

While these studies have compiled a substantial amount of data, in the form of qualitative and quantitative pilot opinion, a fundamental analysis of the effect of the inceptor force-feel system on flight control is found to be lacking. The study of Ref. [6] specifically concluded that a systematic analysis was necessary, since discrepancies with the assigned handling qualities showed that proposed analytical design metrics, or criteria, were not suitable.

\section{Objectives}

The overall goal of the present study is to develop a clearer fundamental understanding of the underlying mechanisms associated with the inceptor dynamics that govern the handling qualities using a manageable analytical methodology.

\section{Approach}

For this purpose an attempt was made at applying an existing analytical pilot model employed in the literature [7] to interpret the handling qualities results from flight and simulation testing. The first step in the study is to perform a detailed parametric variation analysis of the various model components in order to understand their independent effect on the predicted handling qualities. The study then compares the model against results from flight and simulation testing in an attempt to interpret these results and determine its suitability for assessing the handling qualities impact of inceptor force-feel system dynamics.

\section{Analytical Model}

\section{Structural pilot model}

The block diagram shown in Figure 1 illustrates a mathematical representation of the feedback structure proposed by Hess [7], and employed at the core of the analytical model adopted in this study. The model accounts for the fundamental feedback mechanisms, in a simple form, which a human subject relies on for control in a classical regulatory compensatory task. Sub-components for the structural pilot include neuromuscular dynamics $\left(Y_{N M}\right)$, proprioceptive and vestibular feedback ( $Y_{P F}$ and $s K_{\dot{m}}$ respectively), and the visual error compensation $\left(Y_{e}\right)$. Proprioceptive feedback fundamentally accounts for the ability of the pilot model to make corrections to his control inputs based on the perception of stick displacement. Hess [8] argues that this is a key parameter in the prediction of handling qualities. A key feature of this approach is the modeling of the inceptor dynamic response to pilot force inputs $\left(Y_{F S}\right)$ inside of the proprioceptive feedback loop. This allows for the modeling approach to be of use in predicting the handling qualities impact of the inceptor dynamics.

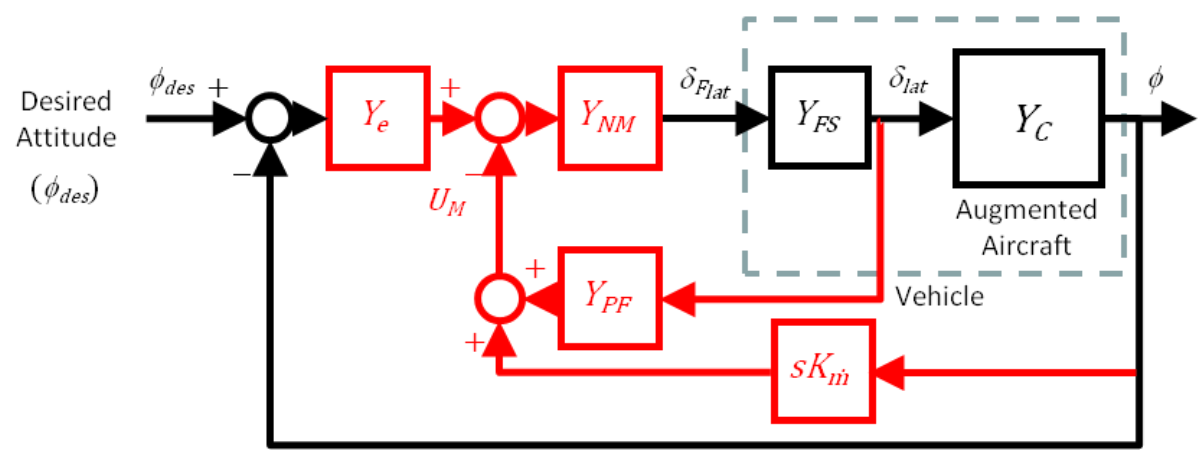

Figure 1: Structural Pilot/Vehicle Model 


\section{Handling Qualities Metric}

The prediction of handling qualities based on this structural pilot model is predicated on the value of the Handling Qualities Metric (HQM)

$$
H Q M=\left|\frac{U_{M}}{\phi_{d e s}}(j \omega) \cdot \frac{1}{K_{e}}\right|
$$

being contained within a prescribed set of boundaries over the typical frequency range of pilot control, i.e., $1-10 \mathrm{rad} / \mathrm{sec}$. The paper will cover this in some detail as there is a significant effect on the appropriate boundaries as a function of the vestibular feedback gain. Here $U_{M}$ is the pilot compensation in response to proprioceptive feedback, and $K_{e}$ is the proportional component of the visual compensation strategy. In essence, the HQM attempts to quantify the 'power' in the compensation that is elicited from the proprioceptive sensory feedback, in response to the attitude commands demanded by a maneuver.

Handling qualities predictions from this model approach can be tailored to be task dependent. This is achieved by specifying the pilot crossover frequency, which effectively serves as an independent tuning parameter to the model. Herein, estimates of the crossover frequency were obtained from an inverse dynamic analysis [8].

\section{Experimental Data}

Validation of the analysis is achieved through comparison with results from flight and simulation tests described in what follows. Testing conducted on the RASCAL JUH-60A and ACT/FHS EC-135 in-flight simulators was described in detail in Ref. [6]. A follow-on pilot-in-the-loop test was conducted in the NASA Ames Vertical Motion Simulator (VMS) as an extension of the flight test study of Reference [6]. Handling qualities ratings and quantitative performance and aircraft state data are available from these tests. The simulation experiment provided a unique opportunity to evaluate the effect of motion cues on the handling qualities predictive criteria and piloted control for the particular set of inceptor force-feel characteristics. Additionally, the trials evaluated an expanded matrix of inceptor damping ratios and natural frequencies, with both a center-stick and side-arm cyclic controllers.

\section{Experimental configurations}

The cyclic inceptor dynamics of [6] maintained the same force displacement gradient of $0.75 \mathrm{lb} /$ in for all configurations. In addition the breakout force was set at $1 \mathrm{lb}$, and a small detent was added for testing with Attitude Command. The experiment tested the various force-feel configurations listed in Table 1 for both Attitude and Rate Command response types.

Table 1: Center-stick force-feel configurations from [6]

\begin{tabular}{ccc}
\hline Configuration & $\begin{array}{c}\text { Bandwidth } \\
(\mathrm{rad} / \mathrm{s})\end{array}$ & Damping Ratio \\
\hline A & 7 & 1.5 \\
B & 23 & 1.5 \\
C & 23 & 0.7 \\
D & 7 & 0.7 \\
F & 9 & 0.9 \\
\hline
\end{tabular}




\section{Evaluation tasks}

Two mission task elements (MTEs) from ADS-33E were used for evaluation of the effects of cyclic force feel characteristics on handling qualities. The two maneuvers are the Hover MTE and the Slalom MTE. The Hover MTE is a low speed maneuver $(6-10 \mathrm{kt})$ that requires the pilot to make small inputs around trim to perform the maneuver. The Slalom MTE is a high speed maneuver (60 kt) that requires the pilot to make low frequency, large amplitude inputs to perform the maneuver. The two tasks are ideally suited to evaluate cyclic force feel characteristics for inputs that are broadly representative of control strategies utilized by helicopter pilots.

\section{Sample Results of Parametric Analysis}

\section{Vestibular feedback}

As shown in Figure 2, the Handling Qualities Sensitivity Function analysis predicts increasingly worsening handling qualities in response to increasing vestibular feedback, based on the theoretical boundaries proposed in the literature [8], as indicated by the progressively larger amplitude peak that develops. This is in stark disagreement with experiment. As was expected, inclusion of motion cues in the simulation testing was found to improve the handling qualities, particularly in the Hover MTE where motion provided a better ability to anticipate the drift of the aircraft. These two results, combined, provide a strong indication that, although arguably validated against fixed base simulation, the theoretical HQ level boundaries are not appropriate for the tasks at hand, where vestibular cuing does seem to play an important role.

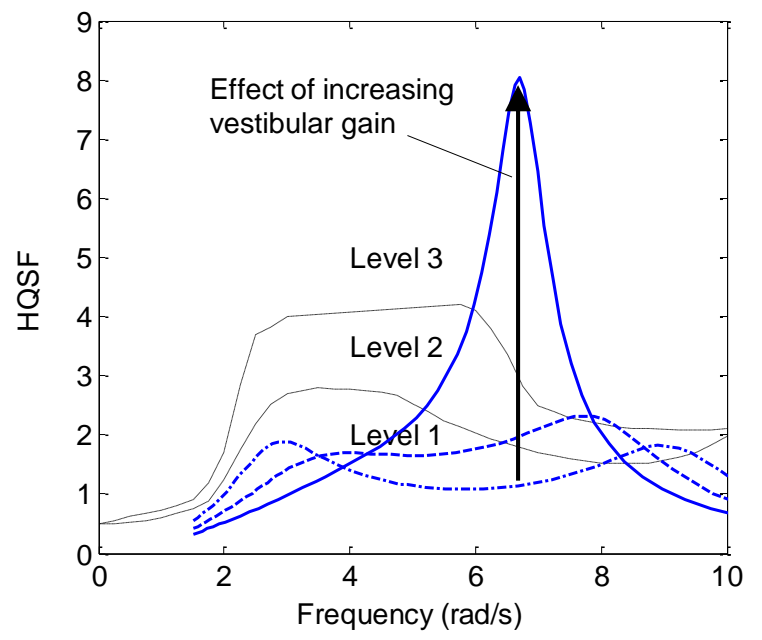

Figure 2: Handling Qualities Sensitivity Function for RC lateral axis Case A (7 rad/s natural frequency and 1.5 damping ratio) and varying vestibular feedback (0,12\% and 25\%). Model configured to $1.4 \mathrm{rad} / \mathrm{s}$ crossover frequency (Slalom).

\section{Inceptor force-feel dynamic characteristics}

A parametric analysis of the effect of inceptor damping and natural frequency in the presence of vestibular feedback was performed for both Rate and Attitude Command response types. Trends predicted by the analytical model were shown to agree with flight and simulation test. Low damping of the inceptor was shown to generate a peak in the handling qualities metric (Figure 3) indicative of potentially degraded handling qualities. 


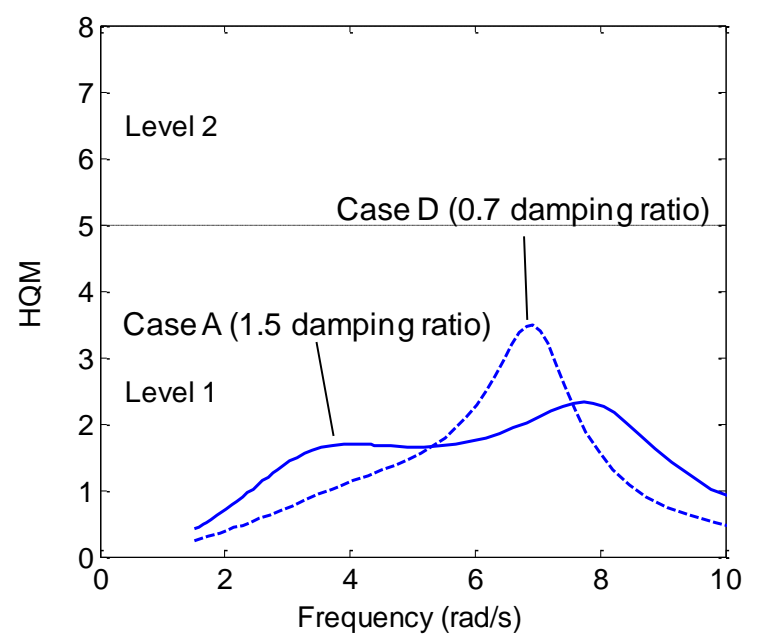

Figure 3: Handling Qualities Metric for RC lateral axis Cases A and D (7 rad/s natural frequency) and $12 \%$ vestibular feedback. Model configured to $1.4 \mathrm{rad} / \mathrm{s}$ crossover frequency (Slalom).

High inceptor natural frequency was found to shift this peak outside of the pilot operating frequency range (Figure 4), where the power of the desired command input is lower, and potentially minimizing the effect of the low damping.

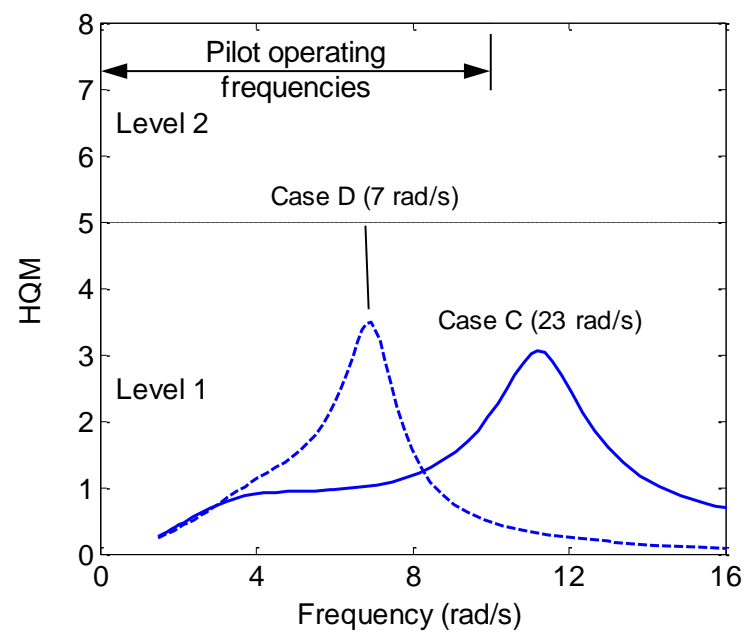

Figure 4: Handling Qualities Metric for Rate Command lateral axis Cases C and D (0.7 damping ratio) and 12\% vestibular feedback. Model configured to $1.4 \mathrm{rad} / \mathrm{s}$ crossover frequency (Slalom).

The combined effect of the two parameters appears to confirm the pilot preference for case B (high damping, high naturally frequency) and the dislike for case D (low damping, low natural frequency) observed in flight for the Hover MTE.

\section{Scope of Paper}

The paper will include additional background on the Handling Qualities Metric and its use, as well as further results from the parametric variation analysis of the pilot vehicle model. Also, a comparison of analysis against flight and simulation data from [6] and the follow-on experiment conducted in the NASA Ames Vertical Motion Simulator in $\mathbf{2 0 1 2}$ will be presented. This latter simulation work has not been presented elsewhere, and therefore, the paper represents an opportunity to publish these results. Finally, the analytical methodology will be assessed to determine its usefulness in refining the handling qualities boundaries for inceptor natural frequency and damping criteria. 


\section{References}

[1] Landis, K., and Glusman, S., "Development of ADOCS controllers and control laws: Vols 1-3". USAAVSCOM TR 84-A-7, March 1987.

[2] Watson, D. C., and Schroeder, J. A. "Effects of Stick Dynamics on Helicopter Flying Qualities," AIAA-90-3477-CP, Presented at the AIAA Guidance, Navigation and Control Conference, August 1990.

[3] Mitchell, D. D., Aponso, B. L., and Klyde, D. H., "Feel Systems and Flying Qualities". AIAA-953425-CP, Presented at the AIAA Atmospheric Flight Mechanics Conference, Baltimore, MD, Aug. 7-10, 1995.

[4] Morgan, M. J., "An Initial Study into the Influence of Control Stick Characteristics on the Handling Qualities of a Fly-By-Wire Helicopter," AGARD-CP-508, February, 1991.

[5] Greenfield, A., and Sahasrabudhe, V., "Side-Stick Force-Feel Parametric Study of a Cargo-Class Helicopter," Proceedings of the 67th annual forum of the American Helicopter Society, Virginia Beach, VA, May 3-5, 2011.

[6] Lusardi, J.A., et al. "In Flight Evaluation of Active Inceptor Force-Feel Characteristics and Handling Qualities,". Proceedings of the 68th Annual Forum of the American Helicopter Society, Phoenix, AZ, May, 2012.

[7] Hess, R. A., "Analyzing Manipulator and Feel System Effects in Aircraft Flight Control," IEEE Transactions on Systems, Man and Cybernetics, Vol. 20, (4), July/August 1990, pp. 923-931.

[8] Hess, R. A., Zeyada, Y., and Heffley, R. K. "Modeling and Simulation for Helicopter Task Analysis," Journal of the American Helicopter Society, Vol. 47, (4), October 2002, pp. 243-252. 